\title{
Nutritional psychiatry: A solution for socioeconomic disparities in access to mental health care?
}

\author{
Caroline Wallace*
}

Centre for Neuroscience Studies, Queen's University, Kingston, ON, Canada

${ }^{\star}$ Author for correspondence (caroline.wallace@queensu.ca)

\begin{abstract}
:
As in all sectors of healthcare, socioeconomic status (SES) affects an individual's ability to benefit from psychiatric care. Mood and anxiety disorders are the most common mental illnesses for which psychiatric care is sought, and while there are options for effective treatments available, they often have accompanying costs. Further to costs, issues with the heterogeneity of mental illness have led resarchers to explore other options for psychiatric care. Nutritional psychiatry is an emerging field that uses dietary and nutritional approaches to target the gut-brain axis for the prevention and treatment of mental illness, including mood and anxiety disorders. Nutritional psychiatry has been promoted as being an advantageous alternative to classic mental health treatments due to its broader accessibility, given the lower costs associated with lifestyle changes than medication and psychotherapy. At a glance this may appear accurate, but upon closer examination, may not be entirely true. Factors surrounding healthy eating, food deserts, the supplement industry, and adherence to lifestyle changes are all barriers present in nutritional psychiatry that are accompanied by additional costs. These costs likely contribute to the disparity between low SES and high SES individuals benefitting from nutritional psychiatry approaches in a similar way to standard treatments. This commentary reviews these factors and suggests that while there are certainly benefits, nutritional psychiatry may not be the accessible treatment option it is purported to be. The importance of clinical researchers' awareness of these disparities in the field is also discussed.
\end{abstract}

It should come as no surprise that socioeconomic inequalities plague our healthcare system and prevent individuals with lower socioeconomic status (SES) from obtaining care. SES continues to affect health even once care is received by limiting the ability to benefit from treatment options. This includes mental health care, with access and costs of treatment being the primary barriers with which patients with mood and anxiety disorders struggle. Mood and anxiety disorders such as depression affect approximately $11 \%$ of Canadians and are the leading disorders for which people seek mental health care [1]. Standard options for the treatment of depression include antidepressant medication and/or psychotherapy. In Canada, antidepressant medication costs between $\$ 30$ and $\$ 200$ per month, and while some counselling and therapy can be provided at no cost within universities or other institutions, private psychotherapy costs between $\$ 50$ and $\$ 220$ per one-hour session. In most cases, these treatments require a prescription from a physician. While most provincial drug plans and formularies cover a portion of the cost for prescription drugs and psychotherapy, there are often outstanding costs still to be paid out of pocket such as dispensing fees.

Depression is a complex psychiatric disorder characterized by both psychological and physiological symptoms that impair daily functioning. Like most mental health disorders, it is highly heterogeneous; the etiology, pathophysiology, symptomatology, and response to treatment vary widely between patients [2]. This has made mood disorders difficult to treat using standard medication. For these reasons, other approaches for alleviating symptoms associated with mood disorders are being explored by researchers, several of which involve targeting the microbiota in the gut. The connection between the brain and the gut was established long ago, as early as the eighteenth century [3]. With recent advances in techniques and an increased appreciation for the depth of the connection, research on the relationship between the brain and the gut has broadened and is now termed the "gut-brain axis" [4].

The gut-brain axis can be targeted to help improve mental health by applying techniques from the field of nutritional psychiatry. Nutritional psychiatry is an emerging field studying how diet and nutrition may be used in the prevention and treatment of mental illness. Nutritional psychiatry can be broken down into three approaches: 1) using specific nutrient supplements, such as vitamins, polyunsaturated fatty acid supplements, and probiotic supplements; 2) consuming functional foods, or foods that are fortified in specific nutri-
This is an Open Access article distributed under the terms of the Creative Commons Attribution License (CC BY 4.0)

License deed can be found at: http://creativecommons.org/licenses/ by/4.0/ https://doi.org/10.29173/hsi288 Received 6 February 2020 Accepted 30 April 2020 
ents; and 3) altering whole dietary patterns. The nutrients we obtain through our diet and supplements alter our gut microbiome. These changes in our gut microbiome affect brain development and function via several interconnected biophysiological pathways encompassing the gut-brain axis, including the autonomic nervous system, the enteric nervous system, the neuroendocrine system, and the immune system [4]. This is true for both healthy and unhealthy eating behaviours. Nutritional psychiatry functions on an evidence-based premise of consuming foods and supplements that, through the gut-brain axis, aid in regulating neurotransmission critical for mood and anxiety regulation. Currently, research indicates that adhering to a Mediterranean-type diet, consisting of plenty of fresh produce, whole grains, lean proteins and healthy fats, may be effective in alleviating mental illness symptoms [5, 6]. Several specific nutrients such as probiotics are under investigation as potential standalone supplements as well [7]. These approaches remain in the exploratory stage and are not yet considered an approved treatment for mood and anxiety disorders, making the prevalence of their clinical application in the field unknown. However, the potential benefits they offer are numerous.

Nutritional psychiatry approaches may be preferential to standard treatments for alleviating mental illness symptoms for several reasons. These approaches do not carry the same stigma common to standard treatments, which often leads to poor treatment adherence. There is also lower risk of contraindications with other medications, making them safer and more tolerable options, and they may be more suitable for people who are reluctant to take medications. Further, they are not accompanied by distressing side effects. In fact, the beneficial effects of nutritional psychiatry approaches may indeed extend into other aspects of physical health and lead to the adoption of further health behaviours.

Given the socioeconomic barriers to standard mental health care, perhaps one of the most appealing aspects of nutritional psychiatry is the idea that anyone, regardless of SES, can reap its benefits. Targeting the gut-brain axis using nutritional psychiatry approaches has been promoted as being a more accessible alternative to standard treatments for mental health disorders, such as medication and psychotherapy. To make nutritional psychiatry-based lifestyle changes, it is not essential to see a specialist or even a general practitioner, nor budget for prescription or therapy costs. Thus, ostensibly there should be no socioeconomic disparity in using nutritional psychiatry approaches to alleviate depressive symptoms. However, this is not invariably true, and this caveat requires serious consideration as nutritional psychiatry becomes increasingly mainstream. While nutritional psychiatry undoubtedly has its benefits, accessibility may not be one of them. There exist several significant socioeconomic barriers to having nutritional psychiatry approaches universally accessible. First, a significant financial investment is required for healthy eating. Second, there is a distinct lack of availability of healthy food in many geographical areas. Third, the supplement industry has driven up costs in response to popular culture trends. Fi- nally, adherence to lifestyle changes such as diet is notoriously difficult. These barriers will be reviewed here and discussed in the context of how nutritional psychiatry does indeed have many benefits, but universal accessibility may not be one of them.

\section{Eating healthy is expensive}

While there is no precise definition of a 'healthy diet', dieticians generally recommend dietary patterns that include a variety of fresh fruits and vegetables, whole grains, lean proteins, and healthy fats. This is consistent with what is being studied and appears promising for mental health $[5,6]$. A 2013 meta-analysis that critically evaluated data from studies across 10 countries found that consuming these healthier foods costs approximately US $\$ 1.50$ more per person per day than eating an unhealthy diet [8]. The costs for basic healthy foods are not the only things that are more expensive: functional foods, or foods that are fortified in a given nutrient are often pricier as well. These costs add up quickly, and grocery stores do not accept prescriptions for healthy eating, nor is there insurance coverage for a filet of salmon and a bundle of kale. To make things worse, these healthy choices are often not only out of reach financially, but geographically as well.

\section{Food deserts persist}

Food deserts are geographical areas with limited access to fresh, healthy foods. This is due to a lack of supermarkets that offer a larger variety of affordable, healthy, and better-quality foods compared to smaller convenience stores that stock more expensive and less healthy prepackaged foods or fast-food chains [9]. Food deserts are also often located in low-SES areas [9]. This is seen most notably and on a large-scale in Northern Canada where there is very limited fresh food supply, and what is available has been documented as being up to $81 \%$ more expensive than the rest of the country [10]. To access healthy foods, those who live in food deserts must have access to a vehicle or pay for public transportation. Since people tend to make food choices based on availability, this leads to individuals in food deserts consuming more unhealthy foods [11], or having to spend more money travelling to access healthier foods. This further adds to the disparity of being able to access nutritional psychiatry-based mental health care approaches. When considering alternatives, longer-lasting and shelf-stable nutritional supplements that may replace vital nutrients are unfortunately not a viable option due to their own drawbacks.

\section{The supplement world is designed to make a profit}

The supplement industry is an ever-growing multi-billion-dollar industry. Nutritional supplements are undeniably effective in the case of a deficiency, such as iron supplements for anemia, or when additional supplementation is required, like folic acid during pregnancy to support the growing fetus 
[12]. However, despite recent evidence suggesting that nutritional supplements like a multivitamin have no added benefit for those who are otherwise healthy [13], the supplement industry has grown as a result of rising popularity in 'wellness culture'. Wellness culture is an industry with good intentions of achieving overall physical, emotional, spiritual, intellectual, and social wellbeing, but one which is plagued by pseudoscience and is driven by profits. A cornerstone of 'wellness culture' is the use of nutritional supplements. It has driven up the cost of supplements, including vitamins, botanicals, minerals, proteins and amino acids, fibers, and specialty carbohydrates, polyunsaturated fatty acids, and more, making it expensive and less accessible to purchase these over-the-counter supplements.

\section{Diet is a hard habit to change}

Nutritional psychiatry approaches are largely based on lifestyle modification. While this may seem like a more viable option than taking a daily medication or attending therapy sessions, maintaining dietary habits is notoriously one of the most difficult lifestyle changes to adhere to. Rates of non-adherence to chronic illness treatments can be as high as $80 \%$, with up to $60 \%$ of patients prematurely abandoning healthy behaviour changes [14]. Individuals who use a diet regimen for a specific health issue, whether it be physical or mental or both, will need to be rigorous with their adherence to get the desired outcome. This may require added expenses: regular follow-up appointments with a healthcare professional, habit-tracking apps on a smartphone, or support groups, to name a few. These additional financial commitments may not be possible for those with low SES.

Given the factors considered above, SES may indeed affect individuals' access to nutritional psychiatry-based approaches just as much as their access to classic treatments for mental illness, limiting their ability to benefit from these approaches. Nutritional psychiatry offers many advantages, and while these approaches are not yet considered an approved and prescribable course of treatment, research continues to advance in this direction. Clinical studies are currently underway examining both the effectiveness of this proposed new approach as well as strategies for treatment. These clinical studies will inform us on how to proceed with the best course of action for intervention, but clinical researchers must also be cognizant of the socioeconomic impact of these interventions. Should the research support nutritional psychiatry as an evidence-based treatment option for mental illness, informed researchers can then help to make recommendations to policy makers for nutritional psychiatry strategies that will reduce associated costs. This will allow those across the socioeconomic spectrum to have equal opportunity to choose a nutritional psychiatry approach to alleviate their symptoms and be able to equally reap the benefits of this proposed new approach to mental health care.

\section{References}

1. Statistics Canada, 2013 Canadian Community Health Survey: Annual Component.

2. Kennedy S, Downar J, Evans K, Feilotter H, Lam R, MacQueen $\mathrm{G}$, et al. The Canadian biomarker integration network in depression (CAN-BIND): advances in response prediction. Curr Pharm Des. 2012 Dec 1;18(36):5976-89. DOI: https://doi. org/10.2174/138161212803523635

3. Miller I. The gut-brain axis: historical reflections. Microb Ecol Health Dis. 2018 Nov 23;29(2):1542921. DOI: https://doi.org/10.108 $0 / 16512235.2018 .1542921$

4. Foster JA, Neufeld KA. Gut-brain axis: how the microbiome influences anxiety and depression. Trends Neurosci. 2013 May 1;36(5):305-12. DOI: https://doi.org/10.1016/j.tins.2013.01.005

5. Jacka FN, O'Neil A, Opie R, Itsiopoulos C, Cotton S, Mohebbi M, et al. A randomised controlled trial of dietary improvement for adults with major depression (the 'SMILES'trial). BMC Med. 2017 Dec;15(1):23. DOI: https://doi.org/10.1186/s12916-017-0791-y

6. Parletta N, Zarnowiecki D, Cho J, Wilson A, Bogomolova S, Villani A, et al. A Mediterranean-style dietary intervention supplemented with fish oil improves diet quality and mental health in people with depression: A randomized controlled trial (HELFIMED). Nutr Neurosci. 2019 Jul 3;22(7):474-87. DOI: https://doi.org/10.1080/102 8415X.2017.1411320

7. Wallace CJ, Foster JA, Soares CN, Milev RV. The effects of probiotics on symptoms of depression: protocol for a double-blind randomized placebo-controlled trial. Neuropsychobiology. 2020;79(1-2):108-16. DOI: https://doi.org/10.1159/000496406

8. Rao M, Afshin A, Singh G, Mozaffarian D. Do healthier foods and diet patterns cost more than less healthy options? A systematic review and meta-analysis. BMJ Open. 2013 Dec 1;3(12):e004277. DOI: 10.1136/bmjopen-2013-004277

9. Walker RE, Keane CR, Burke JG. Disparities and access to healthy food in the United States: A review of food deserts literature. Health \& Place. 2010 Sep 1;16(5):876-84. DOI: https://doi.org/10.1016/j. healthplace.2010.04.013

10. Kenny TA, Fillion M, MacLean J, Wesche SD, Chan HM. Calories are cheap, nutrients are expensive-The challenge of healthy living in Arctic communities. Food Policy. 2018 Oct 1;80:39-54. DOI: https:// doi.org/10.1016/j.foodpol.2018.08.006

11. Furey S, Strugnell C, McIlveen MH. An investigation of the potential existence of "food deserts" in rural and urban areas of Northern Ireland. Agr Hum Values. 2001 Dec 1;18(4):447-57. DOI: https:// doi.org/10.1023/A:1015218502547

12. Greenberg JA, Bell SJ, Guan Y, Yu YH. Folic acid supplementation and pregnancy: more than just neural tube defect prevention. Rev Obstet Gynecol. 2011;4(2):52. DOI: 10.3909/riog0157

13. Guallar E, Stranges S, Mulrow C, Appel LJ, Miller ER. Enough is enough: stop wasting money on vitamin and mineral supplements. Ann Intern Med. 2013 Dec 17;159(12):850-1. DOI: https://doi. org/10.7326/0003-4819-159-12-201312170-00011

14. Middleton KR, Anton SD, Perri MG. Long-term adherence to health behavior change. Am J Lifestyle Med. 2013 Nov;7(6):395-404. DOI: https://doi.org/10.1177/1559827613488867

(C) The Author(s) 